\title{
Social Media Marketing Effectiveness of Non-Profit Organizations: An Analysis on Environmentalist Organizations $^{1}$
}

DOI: $10.26466 /$ opus.918660

*

\section{Zübeyde Yalçın* - Meltem Canoğlu**}

* Graduate St., Osmaniye Korkut Ata University, Institute of Social Sciences, Osmaniye/Turkey E-Posta: zubeydeyalcing@gmail.com ORCID: 0000-0003-2334-7712

** Assist. Prof. Dr., Osmaniye Korkut Ata Uni., Faculty of Eco. and Ad. Scie., Osmaniye/ Turkey E-Posta: meltemcanoglu@osmaniye.edu.tr

ORCID: $\underline{0000-0001-7712-1650}$

\begin{abstract}
Today, all for-profit and non-profit organizations use social media platforms as a marketing tool. Social networking sites such as Facebook, Twitter and Instagram have created a new area for organizations to communicate with their target audience, to increase brand awareness and to provide brand engagement. Measuring social media success for the organization along with the use of social media as a marketing tool has become a significant issue. This study aims to measure the social media marketing effectiveness of non-profit organizations, and thus examining Facebook, Twitter and Instagram accounts of 35 organizations operating in the field of environmental protection. Based upon the data obtained from Facebook, Twitter and Instagram social network sites, the organizations' use of social media in the same event was interpreted according to the social media marketing effectiveness measurement metrics identified by Hoffman and Fodor; moreover, to what extent they were successful were analyzed in terms of brand awareness, brand engagement and word of mouth communication. TEMA Foundation and WWF Foundation were noted to use three social network sites more effectively and they were more successful regarding brand awareness, brand engagement and word of mouth communication compared to the other organizations.
\end{abstract}

Keywords: $\quad$ social media, social networks, social media marketing, efficiency of social media marketing, non-profit organizations

\footnotetext{
${ }^{1}$ This article was produced from Zübeyde YALÇIN's Master Thesis.
} 


\section{Kâr Amacı Gütmeyen Organizasyonların Sosyal Medya Pazarlaması Etkinliği: Çevreci Organizasyonlar Üzerine Bir İnceleme}

Öz

Günümüzde kâr amacı güden ve kâr amacı gütmeyen tüm organizasyonlar sosyal medya platformların bir pazarlama aracı olarak kullanmaktadırlar. Facebook, Twitter ve Instagram gibi sosyal ăg siteleri organizasyonlar için hedef kitle ile iletişim kurmak, marka bilinirliğini arttırmak ve markalarına karşı bir bağlllık sağlamaları için yeni bir ortam oluşturmuştur. Sosyal medyanın pazarlama aracı olarak kullanılması ile birlikte organizasyonlar için sosyal medya başarısını ölçmek önemli bir konu haline gelmiştir. Kâr amacı gütmeyen organizasyonların sosyal medya pazarlaması etkinliğinin ölçülmesi hedeflenen bu çalışmada, çeore koruma alanında faaliyet gösteren 35 organizasyonun Facebook, Twitter ve Instagram hesaplart incelenmiştir. Organizasyonların Facebook, Twitter ve Instagram sosyal ăg sitelerinden elde edilen veriler doğrultusunda sosyal medyayı aynı etkinlikte kullanıp kullanmadıklar Hoffman ve Fodor'un sosyal medya pazarlaması etkinliği ölçme kriterlerine göre yorumlanmıştır ve

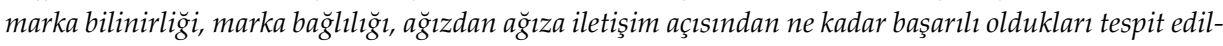
meye çalışılmıştır. TEMA Vakfı ve WWF Vakfi'nın, örneklem çerçevesinde incelenen diğgr organizasyonlara kıyasla üç sosyal medya sitesini daha etkin kullandıkları ve marka bilinirliği, marka bağlllı̆̆ı ve ağızdan ağıza iletiş̧imde daha başarılı oldukları tespit edilmiştir.

Anahtar Kelimeler : sosyal medya, sosyal ağlar, sosyal medya pazarlaması, sosyal medya pazarlaması etkinliği, kâr amacı gütmeyen organizasyonlar 


\section{Introduction}

Great changes in today's economy have shaped the understanding of marketing with the driving force of technology and globalization, and people not only benefit from the advantages of development but they also have to struggle with the emerging disadvantages. The main point to be taken into account is how to act by planning the future while consuming scarce resources. The statement of the Indians, "We do not inherit the earth from our ancestors, we borrow it from our children." summarizes the subject. To give an example, on the one hand, people experience the comfort of the air conditioner against the heat; on the other, they are faced with the global warming effect of air conditioners. On that point, encouraging people to use limited air conditioning against global warming, which is the negative effect of air conditioning can be defined as a significant behavior (Nurtanış Velioğlu and Çoknaz, 2010, p.455).

Besides, social media, another element that has come into being with the development of technology, is used for various purposes such as communication, access to information, entertainment and shopping in today's society. In this regard, social media has become an important tool as it changes individuals' daily lives. Since individuals have the opportunity for producing and consuming the content they wish on social media, they become owners of an informal communication channel in their daily lives. For-profit and non-profit organizations tend towards social media sites as social media users are people. Social media enables mutual communication that is interaction, by discarding the barriers between organizations and the target audience (Saatçioğlu, 2017, p.159).

Thus, non-profit organizations that are willing to benefit from the opportunities offered by the internet and that are aware of new technologies in order to reach their target audiences need to take advantage of the chance to present their functions such as announcing their activities and collecting donations through the internet and new communication technologies (Y1lmazel, 2011, p.2).

This study attempts to identify and interpret the effective use of social media by non-profit organizations operating in the field of environmental and natural life protection that exists on social media sites, considered as 
a way of communicating with people, via social media marketing effectiveness measurement metrics announced by Hoffman and Fodor.

\section{Method}

This study aims at measuring non-profit organizations' effectiveness on social media marketing activities. In this sense, the population of the study consisted of all associations and foundations being active in the environment and wildlife protection areas in Turkey. The sample was determined by judgement (purposive) sampling, one of the non-random sampling methods. Judgement (purposive) sampling is defined as the sampling type in which the decision of the sample is made by the researcher (Zeren, 2015, p.228). Therefore, the sample of the study included associations and foundations for the protection of environment and natural life, which have a website, whose Facebook, Twitter and Instagram accounts are shared on their websites, and which use these three social media tools simultaneously. The websites of the General Directorate of Civil Society Relations (https://www.siviltoplum.gov.tr/, 28.05.2019) and the General Directorate of Foundations (https://www.vgm.gov.tr, 28.05.2019) were used while determining the sample. In addition, the sample of a doctoral thesis (Kaçar, 2014, p.275) was also taken into consideration.

The organizations included in the sample of the study are as follows; TEMA (The Turkish Foundation for Combating Soil Erosion, for Reforestation and the Protection of Natural Habitats), WWF Foundation (World Wildlife Foundation), ÇEVKO Foundation (Environmental Protection and Packaging Waste Recovery and Recycling Foundation), ÇEKÜL (The Foundation for the Protection and Promotion of the Environment and Cultural Heritage), TUDAV Foundation (Turkish Marine Research Foundation), TÜÇEV Foundation (Turkey Environmental Protection Foundation), Mind Your Waste Foundation, Tekfen Foundation, Disaster Awareness Association, DOĞÇEV Association (Nature and Environment Protection, Survival Association), DOSDER Association (Nature and Sustainability Association), Natural Life Association, the Rural Environment Association, SURGED Association (Sustainable Future Association), GÜNDER Association (International Solar Energy Society Turkey), DMAD As- 
sociation (Marine Mammals Research Association), MUKEDER Association (Happy Cats Association), ECORDING Association (Conscious Life Association ), KEŞAN DOÇEK Association (Keşan Nature and Environment, Culture Association), ESÇEVDER Association (Eskişehir Environmental Protection and Development Association), ASPEG Association (Anatolian Speleology Group Association), ÇEKUD Association (Association of Environmental Organizations), TURMEPA Association (DenizTemiz Association), BOĞAZİÇİ PATİLILER Association (Life Animals and Nature Conservation Association), Pan Association, Street Is Ours Association, Earth Association, Mediterranean Conservation Association, Environmentalist Energy Association, Nature Association, Zeytince Association, KOHAYDER Association (Kocaeli Friends of Nature and Animal Association), Hemşin Life Association, TİB Federation (Federation of Trakya Scouts Union), DOHAS Association (Nature and Animal Lovers Association).

The official Facebook, Twitter and Instagram accounts of the non-profit organizations were examined between June 4-11, 2019. In this vein, the dates of the last posts, the number of followers, the number of shares and the user interactions were initially identified from the organizations' social media accounts until June 4, 2019. Since the World Environment Day is June 5, the study was conducted between June 4-11, 2019 (inclusive). The data were interpreted in terms of brand awareness, brand engagement and word-of-mouth communication based on the "Metrics for Measuring Social Media Marketing Effectiveness" developed by Hoffman and Fodor (2010, p. 44) in order to measure the effectiveness of social media marketing. Besides, the rate of being talked about on the social media sites of the organizations was obtained from BoomSocial (https://www.boomsocial.com/), which is one of the free sites used in the measurement of social media marketing effectiveness, and it was evaluated in terms of brand awareness.

This section provides information regarding the metrics for measuring the effectiveness of social media marketing, which is the method of this study. 


\section{Metrics for Measuring Social Media Marketing Effectiveness}

With the emergence and prevalence of new communication technologies, social media tools are widely used in corporate organization and public communication (Luo and Jiang, 2012, p.57). Along with the increasing popularity of social media, the measurement of activities (Luo and Jiang, 2012, p.59) and success have become one of the hottest topics that businesses and organizations using social media platforms are interested in (Barutçu and Tomaş, 2013, p.13). Organizations and businesses must have the ability to monitor social media metrics in order to understand what is posed in the social media environment. There are currently no precise methods for measuring the success of a social media campaign. In this respect, social media marketing effectiveness measurements are a brand new issue that needs to be improved (Cray, 2012, p.46).

It is necessary for an organization to calculate social media marketing effectiveness, gain brand awareness, generate revenue, and determine whether the specified goals such as customer satisfaction have been achieved (https://www.socialbakers.com/blog/7-steps-to-measure -socialmedia-marketing-roi-a-complete-guide, 13.11.2019). Social media sites are used as a marketing tool for most organizations. Traditional methods may not provide concrete results while measuring social media marketing effectiveness (Barutçu and Tomaş, 2013, p.14). Hoffman and Fodor (2010, p.42) noted that the measurement of social media marketing effectiveness is quite unlike from traditional methods, and managers should consider consumer motivations and how they engage in the social media addresses of the brands instead of calculating the returns in terms of customers' feedback while calculating the organization's effectiveness on social media sites.

Hoffman and Fodor (2010, p. 45) emphasized that there are three social media goals that help calculate social media marketing effectiveness; brand awareness, brand engagement and word of mouth. Zarrella, on the other hand, stated that calculating social media marketing effectiveness is much less complicated and it is sufficient to deduct the cost of the social media business (including money and time investments) from the revenue generated to calculate it. He stated that it would be profitable if the result 
was positive, but if not, the campaign addresses should be revisited and attention should be paid to which sites (such as Facebook and Twitter) and tactics created the most value (Wendlandt, 2012, p.18).

Different from Hoffman and Fodor's methods, those developed for measuring social media marketing effectiveness are presented below;

Brand Reach $=$ Facebook Fans + Twitter Followers

Content Reach $=$ Brand Reach $+\sum$ (Shares) $x$ (Access for each Share)

Content Engagement $=$ Posts + Replies $/$ Total Pieces of Social Content

Content Engagement $=$ Clicks $/$ Content Reach

Brand Engagement $=$ Likes + Tweets + Comments + Mentions

Attention $=$ Reach $\times$ Engagement (Wendlandt, 2012, p.26-27; Ghali, 2011, p.4-7).

Table 1 depicts the metrics developed by Hoffman and Fodor (2010, p. $44)$ with a view to measuring social media marketing effectiveness. These metrics include various metrics for social media by classifying them according to social media applications and social media performance targets. Despite not being exhaustive, it is expected to be a useful starting point for organizations to measure the effectiveness of their social media efforts since the metrics listed are easily measured.

Pencarelli and Mele (2018, p.30) highlighted that the higher the interaction value (such as following the page, like-comment) of the organizations' followers with the content published on social media sites is, the higher the value of the organizations' goals (brand awareness, brand engagement, word of mouth) can be achieved. At that point, the data obtained from the social media accounts of the organizations are interpreted in this regard. In a nutshell, this study suggested that the higher the interaction values of organizations such as the number of followers, likes and comments are, the higher the brand awareness, brand engagement and word of mouth communication is. 
Table 1. Hoffman and Fodor's Metrics for Measuring Social Media Marketing Effectiveness

\begin{tabular}{|c|c|c|c|}
\hline $\begin{array}{l}\text { Social Media Ap- } \\
\text { plications }\end{array}$ & Brand Awareness & Brand Engagement & $\begin{array}{l}\text { Word of Mouth Com- } \\
\text { munication }\end{array}$ \\
\hline Blogs & $\begin{array}{l}\text { Number of unique } \\
\text { visits, Number of } \\
\text { return visits, Num- } \\
\text { ber of times book- } \\
\text { marked, Search } \\
\text { ranking }\end{array}$ & $\begin{array}{l}\text { Number of members, } \\
\text { Number of RSS feed } \\
\text { subscribers, Number of } \\
\text { comments, Amount of } \\
\text { user-generated content, } \\
\text { Average length of time } \\
\text { on site, Number of re- } \\
\text { sponses to polls, Con- } \\
\text { tents, Surveys }\end{array}$ & $\begin{array}{l}\text { Number of references to } \\
\text { blog in } \\
\text { other media, Number of } \\
\text { reblogs, Number of } \\
\text { times badge displayed } \\
\text { on other sites, Number } \\
\text { of "likes" }\end{array}$ \\
\hline $\begin{array}{l}\text { Microblogging } \\
\text { (Twitter) }\end{array}$ & $\begin{array}{l}\text { Number of tweets } \\
\text { about the brand, } \\
\text { Valence of tweets } \\
+/-, \text { Number of fol- } \\
\text { lowers }\end{array}$ & $\begin{array}{l}\text { Number of followers, } \\
\text { Number of replies (@) }\end{array}$ & Number of retweets \\
\hline $\begin{array}{l}\text { Social Bookmark- } \\
\text { ing }\end{array}$ & Number of tags & Number of followers, & $\begin{array}{l}\text { Number of additional } \\
\text { taggers }\end{array}$ \\
\hline $\begin{array}{l}\text { Forums, Discus- } \\
\text { sion Boards } \\
\text { (Google Groups) }\end{array}$ & $\begin{array}{l}\text { Number of page } \\
\text { views, Number of } \\
\text { visits, Valence of } \\
\text { posted content }(+,-)\end{array}$ & $\begin{array}{l}\text { Number of relevant top- } \\
\text { ics/threads, Number of } \\
\text { replies, Number of sign- } \\
\text { ups }\end{array}$ & $\begin{array}{l}\text { Incoming links, Cita- } \\
\text { tions in other sites, } \\
\text { Tagging in social book- } \\
\text { marking, Offline refer- } \\
\text { ences to the forum or its } \\
\text { members, In private } \\
\text { communities: number } \\
\text { of } \\
\text { pieces of content (pho- } \\
\text { tos, discussions, } \\
\text { videos); chatter } \\
\text { pointing to } \\
\text { the community } \\
\text { outside of the group, } \\
\text { Number of "likes" }\end{array}$ \\
\hline $\begin{array}{l}\text { Product Reviews } \\
\text { (Amazon) }\end{array}$ & $\begin{array}{l}\text { Number of reviews } \\
\text { posted, Valence of re- } \\
\text { views, Number and va- } \\
\text { lence of other users' re- } \\
\text { sponses to reviews (+/-), } \\
\text { Number of wish list } \\
\text { adds, Number of prod- } \\
\text { ucts included in users' } \\
\text { lists }\end{array}$ & $\begin{array}{l}\text { Length of reviews, } \\
\text { Relevance of re- } \\
\text { views, Valence of } \\
\text { other users' ratings } \\
\text { of } \\
\text { reviews, Number } \\
\text { of wish list adds, } \\
\text { Overall number of } \\
\text { reviewer rating } \\
\text { scores, Average re- } \\
\text { viewer rating score }\end{array}$ & $\begin{array}{l}\text { Number of reviews } \\
\text { posted, Valence of re- } \\
\text { views, Number and va- } \\
\text { lence of other users' re- } \\
\text { sponses to reviews (+/-), } \\
\text { Number of references to } \\
\text { reviews in other sites, } \\
\text { Number of visits to re- } \\
\text { view site page, Number } \\
\text { of times product in- } \\
\text { cluded } \\
\text { in users' lists }\end{array}$ \\
\hline
\end{tabular}


Table 1. (Cont'd) Hoffman and Fodor's Metrics for Measuring Social Media Marketing Effectiveness

\begin{tabular}{|c|c|c|c|}
\hline $\begin{array}{l}\text { Social Media Appli- } \\
\text { cations }\end{array}$ & Brand Awareness & Brand Engagement & $\begin{array}{l}\text { Word of Mouth Com- } \\
\text { munication }\end{array}$ \\
\hline $\begin{array}{l}\text { Social Networks } \\
\text { (Facebook, } \\
\text { LinkedIn) }\end{array}$ & $\begin{array}{l}\text { Number of mem- } \\
\text { bers/fans, Number } \\
\text { of installs of appli- } \\
\text { cation, Number of } \\
\text { impressions, Num- } \\
\text { ber of bookmarks, } \\
\text { Number of re- } \\
\text { views/ratings and } \\
\text { valence +/- }\end{array}$ & $\begin{array}{l}\text { Number of comments, } \\
\text { Number of active users, } \\
\text { Number of "likes" } \\
\text { number of user-gener- } \\
\text { ated items } \\
\text { (photos, threads, re- } \\
\text { plies), Usage metrics of } \\
\text { Applications/ } \\
\text { Widgets, Impressions- } \\
\text { to-interactions ratio, } \\
\text { Rate of activity (how of- } \\
\text { ten members } \\
\text { update their profiles) }\end{array}$ & $\begin{array}{l}\text { Frequency of appear- } \\
\text { ances in } \\
\text { timeline of friends, } \\
\text { Number of posts on } \\
\text { Wall, Number of re- } \\
\text { posts/shares, Number } \\
\text { of responses to friend } \\
\text { referral invites }\end{array}$ \\
\hline $\begin{array}{l}\text { Video and Pho- } \\
\text { tosharing (Flickr, } \\
\text { Youtube, Insta- } \\
\text { gram) }\end{array}$ & $\begin{array}{l}\text { Number of views of } \\
\text { Video/Photo, Va- } \\
\text { lence of video/photo } \\
\text { ratings and com- } \\
\text { ment }(+,-)\end{array}$ & $\begin{array}{l}\text { Number of replies, } \\
\text { Number of page views, } \\
\text { Number of com- } \\
\text { ments/reviews, Number } \\
\text { of subscribers }\end{array}$ & $\begin{array}{l}\text { Number of embed- } \\
\text { dings, Number of in- } \\
\text { coming links, Number } \\
\text { of references in mock- } \\
\text { ups } \\
\text { or derived work, Num- } \\
\text { ber of times republished } \\
\text { in } \\
\text { other social media and } \\
\text { offline, Number of } \\
\text { "likes" }\end{array}$ \\
\hline
\end{tabular}

Source: Hoffman and Fodor, 2010, p. 44

\section{Findings}

This section includes the interpretation of the data elicited by examining the social media accounts of the organizations determined as samples within the scope of the study. The last sharing dates of the organizations, whose social media accounts were initially examined between June 4 and 11, 2019, were identified and depicted in Table 2. The data in Table 2 includes information regarding the sharing date of the organizations until June 4, 2019, when their social media accounts started to be examined. Afterwards, the change in the number of followers was determined according to the dates, and the organizations that gained and lost the most followers were identified. The shares and how the users (consumers) inter- 
acted with these shares were examined. The last sharing dates of the organizations until June 4, 2019 on Facebook, Twitter and Instagram accounts are presented in Table 2 .

Table 2. Last Sharing Dates of Organizations until June 4, 2019

\begin{tabular}{|c|c|c|c|}
\hline $\begin{array}{r}\text { Organizations } \\
\text { Dates }\end{array}$ & Facebook & Twitter & $\begin{array}{r}\text { In- } \\
\text { stagram }\end{array}$ \\
\hline TEMA Foundation & June 3, 2019 & June 3, 2019 & June 3, 2019 \\
\hline WWF Foundation & June 3, 2019 & June 3, 2019 & June 3, 2019 \\
\hline ÇEVKO Foundation & May 31, 2019 & May 31, 2019 & May 31, 2019 \\
\hline ÇEKÜL Foundation & May 31, 2019 & May 31, 2019 & May 31, 2019 \\
\hline TÜDAV Foundation & June 1, 2019 & June 1, 2019 & May 19, 2019 \\
\hline TÜÇEV Foundation & January 4, 2019 & Not shared yet. & Not shared yet. \\
\hline Mind Your Waste Foundation & June 3, 2019 & June 3, 2019 & June 3, 2019 \\
\hline TEKFEN Foundation & $\begin{array}{r}\text { May 29, } \\
2019\end{array}$ & May 25, 2019 & May 29, 2019 \\
\hline Disaster Awareness Association & January 21, 2018 & March 24, 2017 & January 6, 2018 \\
\hline DOĞÇEV Association & June 3, 2019 & May 18, 2019 & June 2, 2019 \\
\hline DOSDER Association & June 3, 2019 & May 21, 2019 & May 27, 2019 \\
\hline Natural Life Association & June 3, 2019 & March 14, 2017 & March 14, 2017 \\
\hline $\begin{array}{l}\text { RURAL ENVIRONMENT Asso- } \\
\text { ciation }\end{array}$ & May 30, 2019 & June 2, 2019 & June 2, 2019 \\
\hline SURGED Association & October 25, 2018 & May 8, 2017 & January 11, 2018 \\
\hline GÜNDER Association & June 2, 2019 & June 2, 2019 & June 2, 2019 \\
\hline DMAD Association & May 31, 2019 & August 28, 2018 & May 21, 2019 \\
\hline MUKEDER Association & May 23, 2019 & May 22, 2019 & May 22, 2019 \\
\hline ECORDING Association & June 1, 2019 & June 1, 2019 & June 1, 2019 \\
\hline KEŞAN DOÇEK Association & June 3, 2019 & April 25, 2019 & May 27, 2019 \\
\hline ESÇEVDER Association & June 3, 2019 & June 3, 2019 & June 3, 2019 \\
\hline ASPEG Association & June 2, 2019 & April 30, 2017 & May 26, 2019 \\
\hline ÇEKUD Association & June 3, 2019 & June 3, 2019 & June 3, 2019 \\
\hline TURMEPA Association & May 27, 2019 & March 6, 2019 & May 31, 2019 \\
\hline $\begin{array}{l}\text { BOĞAZİÇİ PATİLİLER Associa- } \\
\text { tion }\end{array}$ & May 19, 2019 & May 19, 2019 & May 19, 2019 \\
\hline Pan Association & April 3, 2019 & $\begin{array}{r}\text { November 2, } \\
2018\end{array}$ & May 13, 2019 \\
\hline Street is Ours Association & June 3, 2019 & May 30, 2019 & June 3, 2019 \\
\hline Earth Association & May 30, 2019 & May 31, 2019 & May 30, 2019 \\
\hline $\begin{array}{l}\text { Mediterranean Protection Asso- } \\
\text { ciation }\end{array}$ & June 1, 2019 & June 2, 2019 & June 1, 2019 \\
\hline Environmental Energy Association & June 3,2019 & June 3, 2019 & June 3 , 2019 \\
\hline Nature Association & June 2, 2019 & June 2, 2019 & June 2, 2019 \\
\hline Zeytince Association & June 3, 2019 & June 3, 2019 & May 23, 2019 \\
\hline KOHAYDER Association & June 3, 2019 & June 3, 2019 & June 3, 2019 \\
\hline Hemşin Life Association & May 25, 2019 & May 29, 2019 & May 25, 2019 \\
\hline TİB Federation & June 3, 2019 & May 22, 2019 & May 27, 2019 \\
\hline DOHAS Association & June 3, 2019 & May 30, 2019 & June 1, 2019 \\
\hline
\end{tabular}


Table 2 suggests the last sharing contents of the organizations before this study was conducted. Considering their Facebook accounts, Sustainable Future Association (SURGED) and Disaster Awareness Association last made sharing in 2018. They did not produce any content during the week when the study was being carried out. Besides, almost all of the organizations have shared their posts very recently to the date of the review. Moreover, the majority of the organizations continued their shares during the review. The daily number of shares is displayed in the following tables.

Upon analyzing Twitter accounts, Turkey Environment Foundation (TÜÇEV) did not share posts starting from the date of creating the Twitter account until the study was conducted. Likewise, Disaster Awareness Association, Natural Life Association, Sustainable Future Association (SURGED), the Anatolian Speleology Group Association (ASPEG) last made their sharing in 2017, while the Marine Mammal Research Association (DMAD) and the Pan Association enabled sharing in 2018. These above-mentioned organizations did not share any content while the study was being conducted.

Turkey Environment Foundation (TÜÇEV) did not share posts from Instagram account, before and while conducting this study, Natural Life Association finally made sharing in 2017, while Disaster Awareness Association and Sustainable Future Association (SURGED) in 2018.

Hoffman and Fodor's last sharing dates for Facebook social network site are used as a metric in measuring brand engagement. Hoffman and Fodor (2010, p.46) implied that brand engagement can be enhanced through social media platforms in various ways, one of which is to be renewed and updated. They emphasized that visits and views may increase for new and improved blogs. In this regard, it may be wise to mention that the Sustainable Future Association (SURGED) and Disaster Awareness Association were not be able to provide brand engagement since they last shared posts from their Facebook accounts in 2018 and were not active throughout the study.

The data obtained from the organizations' social media accounts also refers to the number of Facebook, Twitter and Instagram followers of the 
organizations. Table 3 contains data on the number of Facebook, Twitter and Instagram followers of the organizations between 4-11 June 2019.

Table 3. The Number of Facebook, Twitter and Instagram Followers of the Organizations between 4-11 June 2019

\begin{tabular}{|c|c|c|c|c|c|c|c|c|c|c|}
\hline $\begin{array}{l}\text { Organ- } \\
\text { iza- } \\
\text { tions }\end{array}$ & 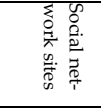 & 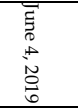 & 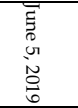 & 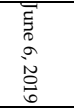 & 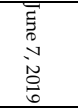 & \begin{tabular}{l}
$\bar{\Xi}$ \\
$\overline{0}$ \\
$\infty$ \\
$i$ \\
\multirow{0}{0}{} \\
\end{tabular} & 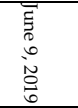 & 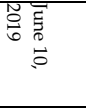 & 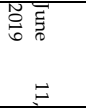 & 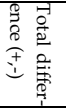 \\
\hline TEMA & Facebook & 575.193 & 575.292 & 575.314 & 575.343 & 575.330 & 575.358 & 575.373 & 575.369 & 176 \\
\hline Foun- & Twitter & 412.656 & 412.796 & 412.841 & 412.860 & 412.890 & 412.920 & 412.962 & 412.989 & 333 \\
\hline dation & Instagram & 234.391 & 234.682 & 234.907 & 235.108 & 235.358 & 235.627 & 235.825 & 236.124 & 1.733 \\
\hline WWF & Facebook & 313.312 & 313.271 & 313.246 & 313.256 & 313.250 & 313.248 & 313.245 & 313.221 & -91 \\
\hline Foun- & Twitter & 264.832 & 264.924 & 264.970 & 265.001 & 265.031 & 265.040 & 265.071 & 265.101 & 269 \\
\hline dation & Instagram & 133.550 & 133.863 & 134.095 & 134.184 & 134.371 & 134.466 & 134.446 & 134.534 & 984 \\
\hline ÇEVK & Facebook & 57.303 & 57.485 & 57.481 & 57.479 & 57.478 & 57.475 & 57.478 & 57.479 & 176 \\
\hline $\mathrm{O}$ & Twitter & 5.903 & 5.915 & 5.917 & 5.920 & 5.919 & 5.927 & 5.931 & 5.935 & 32 \\
\hline $\begin{array}{l}\text { Foun- } \\
\text { dation }\end{array}$ & Instagram & 12.622 & 12.642 & 12.650 & 12.653 & 12.650 & 12.644 & 12.652 & 12.679 & 57 \\
\hline ÇEKÜ & Facebook & 24.637 & 24.635 & 24.636 & 24.634 & 24.634 & 24.635 & 24.643 & 24.654 & 17 \\
\hline $\mathrm{L}$ & Twitter & 7.140 & 7.144 & 7.145 & 7.153 & 7.157 & 7.159 & 7.161 & 7.161 & 21 \\
\hline $\begin{array}{l}\text { Foun- } \\
\text { dation }\end{array}$ & Instagram & 6.151 & 6.152 & 6.164 & 6.168 & 6.175 & 6.181 & 6.188 & 6.194 & 43 \\
\hline TÜ- & Facebook & 5.636 & 5.636 & 5.636 & 5.640 & 5.641 & 5.643 & 5.645 & 5.647 & 11 \\
\hline DAV & Twitter & 753 & 752 & 754 & 753 & 753 & 754 & 754 & 754 & 1 \\
\hline $\begin{array}{l}\text { Foun- } \\
\text { dation }\end{array}$ & Instagram & 580 & 580 & 580 & 580 & 580 & 580 & 580 & 580 & - \\
\hline TÜ- & Facebook & 9 & 9 & 9 & 9 & 9 & 9 & 9 & 9 & - \\
\hline ÇEV & Twitter & 9 & 9 & 9 & 9 & 9 & 9 & 9 & 9 & - \\
\hline $\begin{array}{l}\text { Foun- } \\
\text { dation }\end{array}$ & Instagram & 18 & 18 & 18 & 18 & 18 & 18 & 18 & 18 & - \\
\hline Mind & Facebook & 39.536 & 39.540 & 39.541 & 39.541 & 39.557 & 39.580 & 39.591 & 39.597 & 61 \\
\hline Your & Twitter & 1.655 & 1.684 & 1.685 & 1.684 & 1.685 & 1.690 & 1.691 & 1.699 & 44 \\
\hline $\begin{array}{l}\text { Waste } \\
\text { Foun- } \\
\text { dation }\end{array}$ & Instagram & 12.784 & 12.859 & 12.862 & 12.885 & 12.890 & 12.887 & 12.923 & 12.952 & 168 \\
\hline TEK- & Facebook & 86.349 & 86.350 & 86.348 & 86.351 & 86.347 & 86.370 & 86.393 & 86.420 & 71 \\
\hline FEN & Twitter & 1.686 & 1.686 & 1.688 & 1.688 & 1.690 & 1.691 & 1.692 & 1.693 & 7 \\
\hline $\begin{array}{l}\text { Foun- } \\
\text { dation }\end{array}$ & Instagram & 18.747 & 18.750 & 18.758 & 18.751 & 18.755 & 18.757 & 18.767 & 18.770 & 23 \\
\hline Disas- & Facebook & 632 & 632 & 632 & 632 & 632 & 631 & 631 & 631 & -1 \\
\hline ter & Twitter & 288 & 288 & 288 & 288 & 290 & 290 & 289 & 289 & 1 \\
\hline $\begin{array}{l}\text { Aware } \\
\text { ness } \\
\text { Asso- } \\
\text { ciation }\end{array}$ & Instagram & 263 & 263 & 263 & 263 & 263 & 263 & 263 & 263 & - \\
\hline DOĞÇ & Facebook & 1.407 & 1.407 & 1.407 & 1.407 & 1.405 & 1.404 & 1.404 & 1.404 & -3 \\
\hline EV As- & Twitter & 104 & 104 & 104 & 104 & 104 & 104 & 103 & 102 & -2 \\
\hline $\begin{array}{l}\text { socia- } \\
\text { tion }\end{array}$ & Instagram & 625 & 625 & 626 & 626 & 627 & 625 & 624 & 621 & -4 \\
\hline DOSD & Facebook & 2.318 & 2.324 & 2.326 & 2.332 & 2.336 & 2.343 & 2.345 & 2.355 & 37 \\
\hline ER As- & Twitter & 10 & 10 & 10 & 10 & 10 & 10 & 10 & 10 & - \\
\hline $\begin{array}{l}\text { socia- } \\
\text { tion }\end{array}$ & Instagram & 72 & 72 & 72 & 72 & 74 & 74 & 74 & 74 & 2 \\
\hline Natu- & Facebook & 6.629 & 6.629 & 6.632 & 6.630 & 6.633 & 6.633 & 6.631 & 6.634 & 5 \\
\hline ral Life & Twitter & 572 & 571 & 570 & 570 & 569 & 569 & 570 & 572 & - \\
\hline $\begin{array}{l}\text { Asso- } \\
\text { ciation }\end{array}$ & Instagram & 148 & 147 & 147 & 148 & 148 & 146 & 147 & 147 & -1 \\
\hline RU- & Facebook & 4.780 & 4.783 & 4.783 & 4.794 & 4.802 & 4.807 & 4.813 & 4.821 & 41 \\
\hline RAL & Twitter & 904 & 907 & 906 & 908 & 909 & 909 & 910 & 909 & 5 \\
\hline $\begin{array}{l}\text { ENVI- } \\
\text { RON- } \\
\text { MENT }\end{array}$ & Instagram & 887 & 890 & 889 & 889 & 889 & 891 & 892 & 906 & 19 \\
\hline
\end{tabular}


Social Media Marketing Effectiveness of Non-Profit Organizations: An Analysis on Environmentalist Organizations

\begin{tabular}{|c|c|c|c|c|c|c|c|c|c|c|}
\hline \multicolumn{11}{|l|}{$\begin{array}{l}\text { Asso- } \\
\text { ciation }\end{array}$} \\
\hline SURG & Facebook & 165 & 165 & 165 & 166 & 166 & 166 & 166 & 166 & 1 \\
\hline \multirow{2}{*}{$\begin{array}{l}\text { ED As- } \\
\text { socia- } \\
\text { tion }\end{array}$} & Twitter & 153 & 153 & 152 & 151 & 151 & 151 & 151 & 151 & -2 \\
\hline & Instagram & 312 & 312 & 312 & 311 & 311 & 311 & 311 & 311 & -1 \\
\hline \multirow{3}{*}{$\begin{array}{l}\text { GÜN- } \\
\text { DER } \\
\text { Asso- } \\
\text { ciation }\end{array}$} & Facebook & 5.228 & 5.228 & 5.227 & 5.228 & 5.225 & 5.228 & 5.228 & 5.231 & 3 \\
\hline & Twitter & 5.119 & 5.122 & 5.123 & 5.127 & 5.126 & 5.126 & 5.131 & 5.134 & 15 \\
\hline & Instagram & 794 & 794 & 795 & 793 & 793 & 795 & 797 & 801 & 7 \\
\hline \multirow{3}{*}{$\begin{array}{l}\text { DMA } \\
\text { D As- } \\
\text { socia- } \\
\text { tion } \\
\end{array}$} & Facebook & 1.933 & 1.934 & 1.935 & 1.935 & 1.937 & 1.938 & 1.938 & 1.938 & 5 \\
\hline & Twitter & 30 & 30 & 30 & 30 & 31 & 31 & 31 & 32 & 2 \\
\hline & Instagram & 10.946 & 10.950 & 10.949 & 10.948 & 10.949 & 10.949 & 10.951 & 10.950 & 4 \\
\hline \multirow{4}{*}{$\begin{array}{c}\text { MUKE } \\
\text { DER } \\
\text { Asso- } \\
\text { ciation } \\
\end{array}$} & Facebook & 575 & 581 & 585 & 618 & 629 & 636 & 639 & 640 & 65 \\
\hline & Twitter & 3 & 3 & 3 & 3 & 3 & 3 & 3 & 3 & - \\
\hline & Instagram & 4.663 & 4.634 & 4.640 & 4.640 & 4.639 & 4.641 & 4.655 & 4.657 & -6 \\
\hline & & & & & & & & & & \\
\hline \multirow{3}{*}{$\begin{array}{l}\text { ECOR } \\
\text { DING } \\
\text { Asso- } \\
\text { ciation } \\
\end{array}$} & Facebook & 374 & 374 & 374 & 374 & 374 & 374 & 373 & 373 & -1 \\
\hline & Twitter & 2.973 & 2.972 & 2.970 & 2.966 & 2.963 & 2.968 & 2.967 & 2.967 & -6 \\
\hline & Instagram & 10.752 & 10.752 & 10.746 & 10.725 & 10.709 & 10.698 & 10.696 & 10.694 & -58 \\
\hline \multirow{3}{*}{$\begin{array}{l}\text { KEŞA } \\
\mathrm{N} \\
\text { DOÇE } \\
\text { K As- } \\
\text { socia- } \\
\text { tion }\end{array}$} & Facebook & 5.930 & 5.929 & 5.930 & 5.928 & 5.931 & 5.936 & 5.935 & 5.933 & 3 \\
\hline & Twitter & 111 & 111 & 111 & 111 & 111 & 111 & 111 & 111 & - \\
\hline & Instagram & 1.223 & 1.224 & 1.224 & 1.224 & 1.224 & 1.224 & 1.223 & 1.224 & 1 \\
\hline \multirow{3}{*}{$\begin{array}{l}\text { ESÇEV } \\
\text { DER } \\
\text { Asso- } \\
\text { ciation } \\
\end{array}$} & Facebook & 578 & 582 & 583 & 592 & 597 & 602 & 609 & 611 & 33 \\
\hline & Twitter & 79 & 80 & 79 & 79 & 79 & 79 & 79 & 79 & - \\
\hline & Instagram & 212 & 212 & 212 & 212 & 213 & 213 & 216 & 217 & 5 \\
\hline \multirow{3}{*}{$\begin{array}{l}\text { ASPE } \\
\text { G As- } \\
\text { socia- } \\
\text { tion }\end{array}$} & Facebook & 894 & 894 & 894 & 894 & 894 & 895 & 895 & 895 & 1 \\
\hline & Twitter & 88 & 88 & 88 & 88 & 88 & 88 & 88 & 88 & - \\
\hline & Instagram & 3.380 & 3.376 & 3.378 & 3.373 & 3.369 & 3.370 & 3.371 & 3.365 & -15 \\
\hline \multirow{3}{*}{$\begin{array}{l}\text { ÇEKU } \\
\text { D As- } \\
\text { socia- } \\
\text { tion }\end{array}$} & Facebook & 13.510 & 13.504 & 13.504 & 13.502 & 13.501 & 13.499 & 13.499 & 13.497 & -13 \\
\hline & Twitter & 8.460 & 8.457 & 8.459 & 8.457 & 8.459 & 8.459 & 8.456 & 8.459 & -1 \\
\hline & Instagram & 2.533 & 2.538 & 2.536 & 2.537 & 2.548 & 2.541 & 2.540 & 2.538 & 5 \\
\hline \multirow{3}{*}{$\begin{array}{l}\text { TUR- } \\
\text { MEPA } \\
\text { Asso- } \\
\text { ciation }\end{array}$} & Facebook & 23.573 & 23.574 & 23.570 & 23.572 & 23.569 & 23.567 & 23.562 & 23.557 & -16 \\
\hline & Twitter & 6.875 & 6.873 & 6.869 & 6.868 & 6.869 & 6.870 & 6.885 & 6.890 & 25 \\
\hline & Instagram & 5.195 & 5.204 & 5.215 & 5.222 & 5.270 & 5.277 & 5.291 & 5.365 & 170 \\
\hline \multirow{4}{*}{$\begin{array}{l}\text { BOĞA } \\
\text { ZIÇİ } \\
\text { PATİL } \\
\text { İLER } \\
\text { Asso- } \\
\text { ciation }\end{array}$} & Facebook & 2.499 & 2.497 & 2.496 & 2.496 & 2.496 & 2.497 & 2.497 & 2.497 & -2 \\
\hline & Twitter & 161 & 161 & 161 & 161 & 161 & 161 & 161 & 161 & - \\
\hline & Instagram & 1.552 & 1.549 & 1.548 & 1.548 & 1.548 & 1.546 & 1.544 & 1.545 & -7 \\
\hline & & & & & & & & & & \\
\hline \multirow{3}{*}{$\begin{array}{l}\text { Pan } \\
\text { Asso- } \\
\text { ciation }\end{array}$} & Facebook & 625 & 625 & 625 & 625 & 624 & 623 & 623 & 622 & -3 \\
\hline & Twitter & 40 & 40 & 40 & 40 & 40 & 40 & 40 & 40 & - \\
\hline & Instagram & 1.194 & 1.191 & 1.190 & 1.190 & 1.189 & 1.186 & 1.185 & 1.184 & -10 \\
\hline \multirow{3}{*}{$\begin{array}{l}\text { Street } \\
\text { is Ours } \\
\text { Asso- } \\
\text { ciation }\end{array}$} & Facebook & 3.293 & 3.294 & 3.292 & 3.291 & 3.291 & 3.291 & 3.291 & 3.292 & -1 \\
\hline & Twitter & 3.355 & 3.353 & 3.352 & 3.350 & 3.351 & 3.353 & 3.353 & 3.352 & -3 \\
\hline & Instagram & 1.385 & 1.387 & 1.387 & 1.388 & 1.383 & 1.381 & 1.381 & 1.383 & -2 \\
\hline \multirow{3}{*}{$\begin{array}{l}\text { Earth } \\
\text { Asso- } \\
\text { ciation } \\
\end{array}$} & Facebook & 26.643 & 26.643 & 26.643 & 26.640 & 26.637 & 26.635 & 26.632 & 26.633 & -10 \\
\hline & Twitter & 20.809 & 20.824 & 20.836 & 20.858 & 20.862 & 20.884 & 20.902 & 20.908 & 99 \\
\hline & Instagram & 9.010 & 9.032 & 9.040 & 9.136 & 9.181 & 9.206 & 9.228 & 9.248 & 238 \\
\hline \multirow{3}{*}{$\begin{array}{l}\text { Medi- } \\
\text { terra- } \\
\text { nean } \\
\text { Protec- } \\
\text { tion }\end{array}$} & Facebook & 2.977 & 2.978 & 2.979 & 2.980 & 2.981 & 2.981 & 2.982 & 2.981 & 4 \\
\hline & Twitter & 488 & 489 & 490 & 489 & 489 & 491 & 491 & 492 & 4 \\
\hline & Instagram & 828 & 833 & 861 & 882 & 895 & 895 & 897 & 900 & 72 \\
\hline
\end{tabular}




\begin{tabular}{|c|c|c|c|c|c|c|c|c|c|c|}
\hline \multicolumn{11}{|l|}{$\begin{array}{l}\text { Asso- } \\
\text { ciation }\end{array}$} \\
\hline \multirow{5}{*}{$\begin{array}{l}\text { Envi- } \\
\text { ron- } \\
\text { mental } \\
\text { Energy } \\
\text { Asso- } \\
\text { ciation }\end{array}$} & Facebook & 1.998 & 1.998 & 1.999 & 1.999 & 1.999 & 1.999 & 1.999 & 2.000 & 2 \\
\hline & Twitter & 2.344 & 2.344 & 2.345 & 2.345 & 2.345 & 2.345 & 2.348 & 2.348 & 4 \\
\hline & Instagram & 941 & 940 & 940 & 938 & 935 & 935 & 935 & 939 & -2 \\
\hline & & & & & & & & & & \\
\hline & & & & & & & & & & \\
\hline \multirow{3}{*}{$\begin{array}{l}\text { Nature } \\
\text { Asso- } \\
\text { ciation }\end{array}$} & Facebook & 175.184 & 175.153 & 175.151 & 175.141 & 175.134 & 175.142 & 175.135 & 175.117 & -67 \\
\hline & Twitter & 90.597 & 90.624 & 90.670 & 90.711 & 90.743 & 90.783 & 90.791 & 90.809 & 212 \\
\hline & Instagram & 31.985 & 32.013 & 32.045 & 32.076 & 32.095 & 32.108 & 32.105 & 32.131 & 146 \\
\hline \multirow{3}{*}{$\begin{array}{l}\text { Zeytin } \\
\text { ce As- } \\
\text { socia- } \\
\text { tion }\end{array}$} & Facebook & 2.447 & 2.451 & 2.452 & 2.455 & 2.457 & 2.458 & 2.459 & 2.459 & 12 \\
\hline & Twitter & 267 & 266 & 266 & 266 & 264 & 267 & 267 & 268 & 2 \\
\hline & Instagram & 699 & 700 & 700 & 700 & 701 & 701 & 701 & 701 & 2 \\
\hline \multirow{4}{*}{$\begin{array}{l}\text { KO- } \\
\text { HAY- } \\
\text { DER } \\
\text { Asso- } \\
\text { ciation }\end{array}$} & Facebook & 11.370 & 11.369 & 11.370 & 11.390 & 11.385 & 11.382 & 11.384 & 11.384 & 14 \\
\hline & Twitter & 429 & 429 & 427 & 428 & 429 & 430 & 429 & 428 & -1 \\
\hline & Instagram & 2.241 & 2.244 & 2.248 & 2.244 & 2.246 & 2.246 & 2.242 & 2.251 & 10 \\
\hline & & & & & & & & & & \\
\hline \multirow{3}{*}{$\begin{array}{l}\text { Hemşi } \\
n \text { Life } \\
\text { Asso- } \\
\text { ciation }\end{array}$} & Facebook & 3.019 & 3.018 & 3.018 & 3.017 & 3.016 & 3.022 & 3.027 & 3.032 & 13 \\
\hline & Twitter & 365 & 364 & 363 & 363 & 364 & 364 & 372 & 373 & 8 \\
\hline & Instagram & 792 & 791 & 791 & 790 & 791 & 791 & 793 & 793 & 1 \\
\hline \multirow{3}{*}{$\begin{array}{l}\text { Tí } \\
\text { Feder- } \\
\text { ation }\end{array}$} & Facebook & 1.893 & 1.894 & 1.892 & 1.892 & 1.893 & 1.893 & 1.893 & 1.892 & -1 \\
\hline & Twitter & 142 & 142 & 142 & 142 & 142 & 142 & 142 & 142 & - \\
\hline & Instagram & 487 & 488 & 487 & 487 & 489 & 490 & 488 & 488 & 1 \\
\hline \multirow{3}{*}{$\begin{array}{l}\text { DO- } \\
\text { HAS } \\
\text { Asso- } \\
\text { ciation }\end{array}$} & Facebook & 7.293 & 7.293 & 7.291 & 7.297 & 7.305 & 7.306 & 7.325 & 7.329 & 36 \\
\hline & Twitter & 472 & 472 & 472 & 470 & 470 & 469 & 469 & 468 & -4 \\
\hline & Instagram & 3.569 & 3.569 & 3.559 & 3.555 & 3.547 & 3.552 & 3.547 & 3.543 & -26 \\
\hline
\end{tabular}

Table 3 demonstrates the number of Facebook, Twitter and Instagram followers of the organizations between 4-11 June 2019. Table 3 details about the number of followers in organizations' Facebook accounts as follows: increases and decreases were initially observed in the number of Turkey Environment Foundation (TÜÇEV) followers. In addition, the foundations having the most increasing followers (increased by the same number) were TEMA Foundation and ÇEVKO Foundation with 176 followers. The most losing followers belonged to WWF Foundation with 91 followers and Nature Association with 67 followers.

TEMA Foundation, WWF Foundation, ÇEVKO Foundation, TEKFEN Foundation and Nature Foundation had quite a large number of followers compared to the others. Hoffman and Fodor noted that brand awareness and brand engagement are measured according to the number of followers. In the social media environment, using an application designed by a person on behalf of the company or subscribing to a page created by the organization increases brand awareness (Hoffman and Fodor, 2010, p.45). In this respect, TEMA Foundation, WWF Foundation, ÇEVKO Foundation, TEKFEN Foundation and Nature Association, having the highest 
number of followers, can be said to gain more brand awareness and engagement compared to the other organizations.

As is seen in Table 3, the rate of most talked about TEMA Foundation with the highest number of followers during the present study (4-11 June 2019) is displayed in Figure 1.

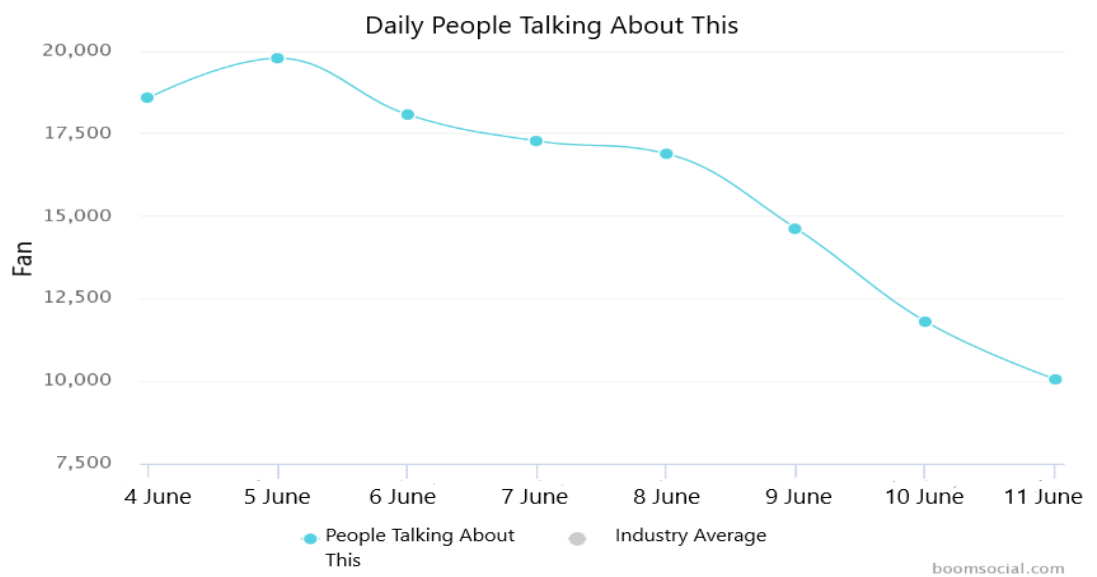

Figure 1. Number of people talking about TEMA Foundation daily (Facebook) (Source: https://www.boomsocial.com/, 12.10.2019)

Having the highest number of followers and increase, TEMA Foundation was also most talked about organization on Facebook social network site. Due to the World Environment Day on June 5, 2019, it became most talked about this week, and 19,766 people talked about TEMA Foundation. When evaluated in terms of brand awareness, TEMA Foundation can be said to be the most known organization among all organizations. Figure 2 shows TEMA Foundation's number of daily engagement on the Facebook social network site. 


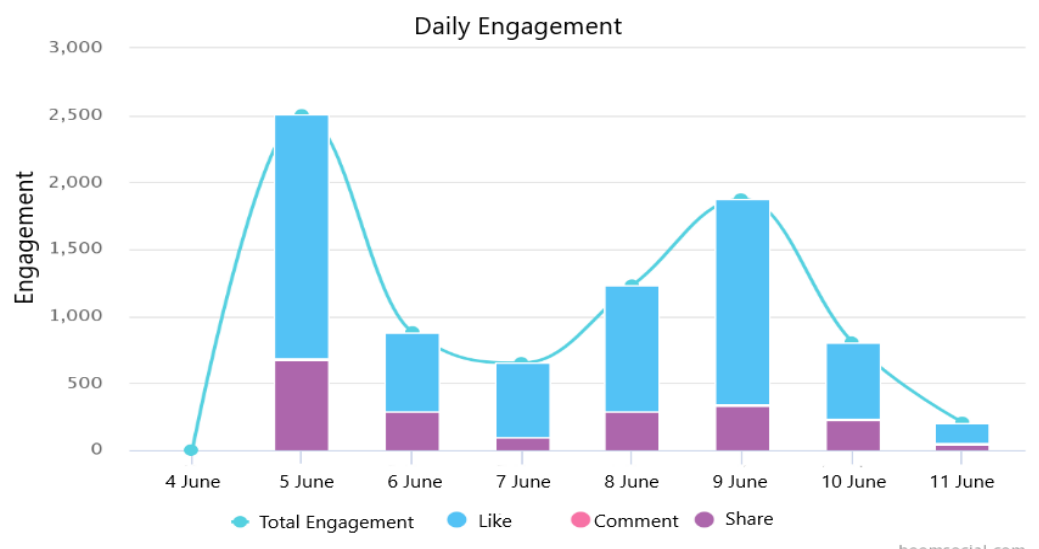

Figure 2. The number of TEMA Foundation's daily engagement (Facebook) (Source: https://www.boomsocial.com/, 12.10.2019)

TEMA Foundation formed the most interactions on June 5, 2019 with 2.503 interactions. Three types of interaction can be made on Facebook. These were; like, comment and share. TEMA Foundation received 1,815 of these engagement types as likes, 9 as comments and 679 as shares. Users mostly preferred likes as the type of engagement.

Table 3 illustrates that the organizations with the highest number of followers on the Twitter social network site were TEMA Foundation, WWF Foundation, Nature Association and Earth Association. Meanwhile, TEMA Foundation, WWF Foundation, Nature Association and Earth Association were the organizations that experienced the highest increase in followers. DOĞÇEV Association, SURGED Association, ECORDING Association, ÇEKUD Association, Street is Ours Association, KOHAYDER Association and DOHAS Association lost followers. TEMA Foundation, WWF Foundation, Nature Association and Earth Association, which had the most followers for the Twitter social network site in terms of brand awareness and brand engagement and gained the most followers during the study, were more known than other organizations and they created engagement to their brands.

The number of daily engagement regarding the Twitter account of TEMA Foundation is depicted in Figure 3. 


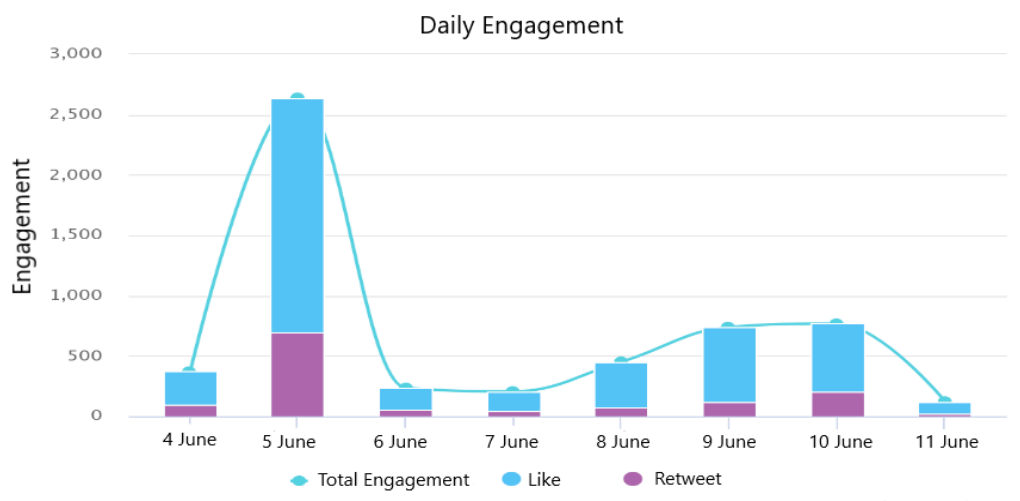

Figure 3. The number of TEMA Foundation's daily engagement (Twitter) (Source: https://www.boomsocial.com/, 12.10.2019)

TEMA Foundation also got the most engagement from its Twitter account on June 5, 2019. Among the engagement types, likes were 4.221 and Retweets were 1.311. Users mostly interacted with likes.

Consumers are in a position to communicate their ideas to other consumers when they generate commitment to brands and when the brand is noticeable to consumers. Thus, satisfied and loyal customers can express their opinions through the social network applications created by the brand itself (Hoffman and Fodor, 2010, p.46). Hoffman and Fodor stated that the effectiveness of word-of-mouth communication could be measured by the number of Retweets. Retweet means reposting the message (tweet) of a person or organization that a user follows to share with their followers (Brown, 2012, p.135). In other words, Twitter users can quote (retweet) a message to convey to their Twitter followers (Ghali, 2011, p.12). TEMA Foundation was most talked about organization on Twitter this week. One of the major reasons for the increase in the number of followers is talking about it and it is successful in word-of-mouth communication.

As observed in Table 3, the two organizations with the highest number of followers on the Instagram social network site were TEMA Foundation and WWF Foundation. Besides, TEMA Foundation and WWF Foundation were those experiencing the highest increase in followers while the study was being conducted. TEMA Foundation had the greatest increase in the number of followers compared to other organizations, with a total of 1,733 
followers. WWF Foundation had the second biggest increase with 984 followers. ECORDING Association was the organization that lost the most followers with 58 followers.

Measuring the effectiveness of brand engagement for video and photo sharing sites like Instagram depends on the number of followers. In addition, the number of comments / review of the posts is regarded as the indicator. TEMA Foundation and WWF Foundation can be mentioned to gained engagement towards their brands thanks to having the highest number of followers. It is possible to measure word-of-mouth communication through the number of likes made by the users to the posts. The number of comments / reviews and likes of is presented in Figure 4 for TEMA Foundation and in Figure 5 for WWF Foundation with graphics.

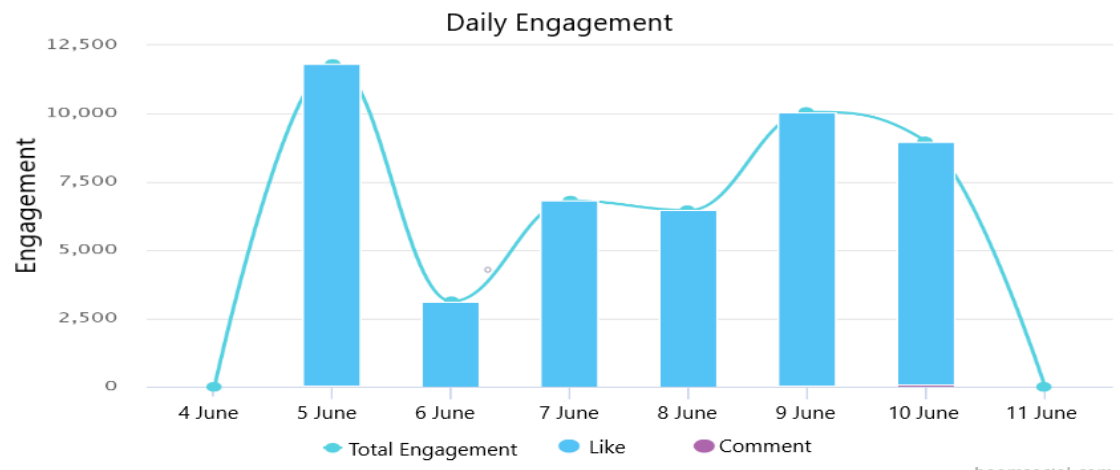

Figure 4. TEMA Foundation's daily engagement (Instagram) (Source: https://www.boomsocial.com/, 12.10.2019)

The daily engagement graph of TEMA Foundation in Figure 4 demonstrates that the foundation enjoyed the most engagement through likes. Likes were mostly made on June 5, 2019 with 11,753 likes, while the comments were on June 10 with 97 comments. TEMA Foundation received a total of 47,008 likes during the current study. Upon examining in terms of word of mouth communication, the engagement was mostly observed in Instagram. Therefore, this may be one of the most significant reasons for the increase in followers. Because users play a role in the social network accounts of brands to reach other users as they express their attitudes towards the brand through social media applications thanks to word-ofmouth communication. In this way, there is an increase in followers 


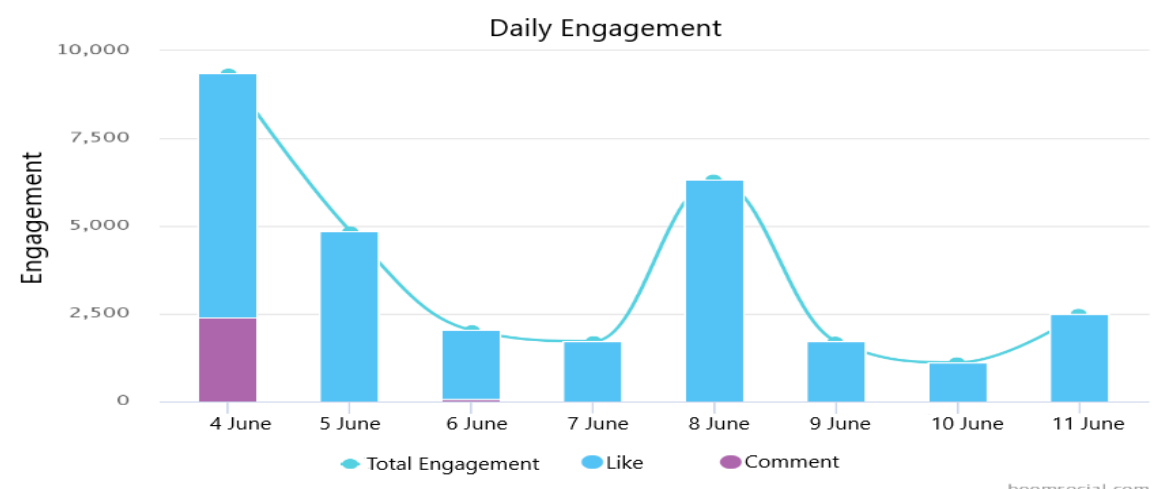

Figure 5. WWF Foundation daily engagement (Instagram) (Source: https://www.boomsocial.com/, 12.10.2019)

Figure 5 shows that the WWF Foundation was the organization that received the most comments from Instagram account this week. 2,377 comments were received as a holiday greeting message in total since June 4, 2019 coincided with the Eid al-Fitr. Brand engagement effectiveness for Instagram is measured according to the number of comments received by the organization. Therefore, it may be wise to emphasize that WWF Foundation has brand engagement and constantly renews and updates its account, and thus, the number of followers is increasing.

The data obtained from the social media accounts is about the number of shares the organizations have made from their social media accounts. Table 4 presents data on the number of Facebook, Twitter and Instagram shares of the organizations between 4-11 June 2019.

Table 4. Number of Facebook, Twitter and Instagram Shares of the Organizations between 4-11 June 2019

\begin{tabular}{|c|c|c|c|c|c|c|c|c|c|c|}
\hline $\begin{array}{l}\text { Organiza- } \\
\text { tions }\end{array}$ & 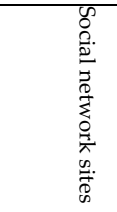 & & 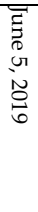 & 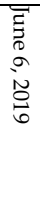 & 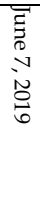 & 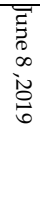 & $\begin{array}{l}\bar{\Xi} \\
\overrightarrow{0} \\
0 \\
N \\
0 \\
0 \\
\sigma\end{array}$ & $\begin{array}{l}\bar{\Xi} \\
\Xi \\
0 \\
0 \\
N \\
0 \\
0\end{array}$ & 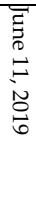 & $\begin{array}{l}\overrightarrow{0} \\
\stackrel{0}{2} \\
\stackrel{0}{0} \\
\overrightarrow{0} \\
\overrightarrow{0}\end{array}$ \\
\hline \multirow{3}{*}{$\begin{array}{l}\text { TEMA } \\
\text { Foundation }\end{array}$} & Facebook & - & 2 & 1 & 2 & 1 & 2 & 2 & 1 & 11 \\
\hline & Twitter & 1 & 6 & 1 & 2 & 1 & 2 & 2 & 1 & 16 \\
\hline & Instagram & - & 3 & 1 & 2 & 1 & 2 & 2 & - & 11 \\
\hline
\end{tabular}




\begin{tabular}{|c|c|c|c|c|c|c|c|c|c|c|}
\hline \multirow{3}{*}{$\begin{array}{l}\text { WWF } \\
\text { Foundation }\end{array}$} & Facebook & 1 & 2 & 1 & 1 & 2 & 1 & 1 & 1 & 11 \\
\hline & Twitter & 1 & 10 & 1 & - & 3 & 1 & 1 & 1 & 18 \\
\hline & Instagram & 2 & 2 & 1 & 1 & 2 & 1 & 1 & 1 & 11 \\
\hline \multirow{3}{*}{$\begin{array}{l}\text { ÇEVKO } \\
\text { Foundation }\end{array}$} & Facebook & - & 2 & - & - & - & - & - & 1 & 3 \\
\hline & Twitter & - & 1 & - & - & - & - & - & 1 & 2 \\
\hline & Instagram & 1 & 1 & - & - & - & - & 1 & 1 & 4 \\
\hline \multirow{3}{*}{$\begin{array}{l}\text { ÇEKÜL } \\
\text { Foundation }\end{array}$} & Facebook & - & 1 & - & - & - & - & - & 1 & 2 \\
\hline & Twitter & - & 1 & - & - & - & - & - & 1 & 2 \\
\hline & Instagram & - & 1 & - & - & - & - & - & - & 1 \\
\hline \multirow{3}{*}{$\begin{array}{l}\text { TÜDAV } \\
\text { Foundation }\end{array}$} & Facebook & - & 1 & - & - & 1 & - & - & 1 & 3 \\
\hline & Twitter & - & 1 & - & - & 1 & - & - & - & 2 \\
\hline & Instagram & - & - & - & - & - & - & - & - & - \\
\hline \multirow{3}{*}{$\begin{array}{l}\text { TÜÇEV } \\
\text { Foundation }\end{array}$} & Facebook & - & - & - & - & - & - & - & - & - \\
\hline & Twitter & - & - & - & - & - & - & - & - & - \\
\hline & Instagram & - & - & - & - & - & - & - & - & - \\
\hline \multirow{3}{*}{$\begin{array}{l}\text { Mind Your } \\
\text { Waste } \\
\text { Foundation }\end{array}$} & Facebook & 1 & 1 & - & 1 & - & 1 & 1 & - & 5 \\
\hline & Twitter & 1 & 1 & - & 1 & - & 1 & 3 & - & 7 \\
\hline & Instagram & 1 & 1 & - & 1 & - & 1 & 1 & - & 5 \\
\hline \multirow{3}{*}{$\begin{array}{l}\text { TEKFEN } \\
\text { Foundation }\end{array}$} & Facebook & 2 & 1 & - & - & - & - & - & - & 3 \\
\hline & Twitter & 1 & 1 & - & - & - & - & - & - & 2 \\
\hline & Instagram & 1 & 1 & - & - & - & - & - & - & 2 \\
\hline \multirow{3}{*}{$\begin{array}{l}\text { Disaster } \\
\text { Awareness } \\
\text { Association }\end{array}$} & Facebook & - & - & - & - & - & - & - & - & - \\
\hline & Twitter & - & - & - & - & - & - & - & - & - \\
\hline & Instagram & - & - & - & - & - & - & - & - & - \\
\hline \multirow{3}{*}{$\begin{array}{l}\text { DOĞÇEV } \\
\text { Association }\end{array}$} & Facebook & 8 & - & 1 & - & 18 & 1 & - & - & 28 \\
\hline & Twitter & - & - & - & - & 13 & - & - & - & 13 \\
\hline & Instagram & - & - & - & - & - & - & - & - & - \\
\hline \multirow{3}{*}{$\begin{array}{l}\text { DOSDER } \\
\text { Association }\end{array}$} & Facebook & 6 & 5 & 6 & 5 & 7 & 7 & 3 & 3 & 42 \\
\hline & Twitter & - & - & - & - & - & - & - & - & - \\
\hline & Instagram & - & 1 & - & - & - & - & - & - & 1 \\
\hline \multirow{3}{*}{$\begin{array}{l}\text { Natural } \\
\text { Life Associ- } \\
\text { ation }\end{array}$} & Facebook & 2 & - & - & 4 & 1 & 8 & 4 & 6 & 25 \\
\hline & Twitter & - & - & - & - & - & - & - & - & - \\
\hline & Instagram & - & - & - & - & - & - & - & - & - \\
\hline \multirow{3}{*}{$\begin{array}{l}\text { RURAL } \\
\text { ENVIRON- } \\
\text { MENT As- } \\
\text { sociation }\end{array}$} & Facebook & - & - & - & 1 & - & - & - & - & 1 \\
\hline & Twitter & - & - & - & - & - & - & - & 1 & 1 \\
\hline & Instagram & - & - & - & 1 & - & - & - & 1 & 2 \\
\hline \multirow{3}{*}{$\begin{array}{l}\text { SURGED } \\
\text { Association }\end{array}$} & Facebook & - & - & - & - & - & - & - & - & - \\
\hline & Twitter & - & - & - & - & - & - & - & - & - \\
\hline & Instagram & - & - & - & - & - & - & - & - & - \\
\hline \multirow{3}{*}{$\begin{array}{l}\text { GÜNDER } \\
\text { Association }\end{array}$} & Facebook & - & - & - & - & - & - & 3 & - & 3 \\
\hline & Twitter & - & - & - & - & - & - & 3 & - & 3 \\
\hline & Instagram & - & - & - & - & - & - & 3 & 1 & 4 \\
\hline \multirow{3}{*}{$\begin{array}{l}\text { DMAD As- } \\
\text { sociation }\end{array}$} & Facebook & - & 1 & - & - & - & - & - & - & 1 \\
\hline & Twitter & - & - & - & - & - & - & - & - & - \\
\hline & Instagram & - & - & 1 & - & - & - & - & - & 1 \\
\hline \multirow{3}{*}{$\begin{array}{l}\text { MUKEDER } \\
\text { Association }\end{array}$} & Facebook & 1 & - & - & - & 3 & - & 2 & - & 6 \\
\hline & Twitter & 1 & - & - & - & - & - & 2 & - & 3 \\
\hline & Instagram & 1 & - & - & - & - & - & 2 & - & 3 \\
\hline
\end{tabular}


Social Media Marketing Effectiveness of Non-Profit Organizations: An Analysis on Environmentalist Organizations

\begin{tabular}{|c|c|c|c|c|c|c|c|c|c|c|}
\hline \multirow{3}{*}{$\begin{array}{l}\text { ECORD- } \\
\text { ING Asso- } \\
\text { ciation }\end{array}$} & Facebook & 1 & - & - & - & - & - & 1 & 1 & 3 \\
\hline & Twitter & 1 & 2 & - & - & - & - & 2 & 1 & 6 \\
\hline & Instagram & 1 & 1 & - & - & - & - & 1 & 1 & 4 \\
\hline \multirow{3}{*}{$\begin{array}{l}\text { KEŞAN } \\
\text { DOÇEK } \\
\text { Association }\end{array}$} & Facebook & - & 1 & - & 3 & 2 & - & 8 & 2 & 16 \\
\hline & Twitter & - & - & - & - & - & - & - & - & - \\
\hline & Instagram & - & - & - & - & - & - & - & 1 & 1 \\
\hline \multirow{3}{*}{$\begin{array}{l}\text { ESÇEVDER } \\
\text { Association }\end{array}$} & Facebook & 1 & 2 & 2 & - & 1 & 1 & - & - & 7 \\
\hline & Twitter & - & 1 & - & 1 & - & 1 & - & - & 3 \\
\hline & Instagram & - & 1 & - & 1 & - & 1 & - & - & 3 \\
\hline \multirow{3}{*}{$\begin{array}{l}\text { ASPEG As- } \\
\text { sociation }\end{array}$} & Facebook & - & - & - & - & 2 & - & - & 1 & 3 \\
\hline & Twitter & - & - & - & - & - & - & - & - & - \\
\hline & Instagram & 1 & - & - & - & - & - & - & 1 & 2 \\
\hline \multirow{3}{*}{$\begin{array}{l}\text { ÇEKUD } \\
\text { Association }\end{array}$} & Facebook & - & 1 & - & - & 1 & - & - & 1 & 3 \\
\hline & Twitter & - & 1 & - & - & 1 & - & 1 & 1 & 4 \\
\hline & Instagram & - & 1 & - & - & 1 & - & 1 & 1 & 4 \\
\hline \multirow{3}{*}{$\begin{array}{l}\text { TURMEPA } \\
\text { Association }\end{array}$} & Facebook & - & - & - & - & - & - & 5 & - & 5 \\
\hline & Twitter & - & - & - & - & - & - & - & - & - \\
\hline & Instagram & 1 & 1 & - & - & 1 & - & 5 & - & 8 \\
\hline \multirow{3}{*}{$\begin{array}{l}\text { BOĞAZİÇİ } \\
\text { PATİLILER } \\
\text { Association }\end{array}$} & Facebook & 2 & - & - & - & - & - & - & - & 2 \\
\hline & Twitter & 2 & - & - & - & - & - & - & - & 2 \\
\hline & Instagram & 2 & - & - & - & - & - & - & - & 2 \\
\hline \multirow{3}{*}{$\begin{array}{l}\text { Pan Associ- } \\
\text { ation }\end{array}$} & Facebook & - & - & - & - & - & - & - & - & - \\
\hline & Twitter & - & - & - & - & - & - & - & - & - \\
\hline & Instagram & - & - & - & - & - & - & - & - & - \\
\hline \multirow{3}{*}{$\begin{array}{l}\text { Street is } \\
\text { Ours Asso- } \\
\text { ciation }\end{array}$} & Facebook & - & - & - & - & - & - & - & - & - \\
\hline & Twitter & - & 2 & - & - & - & - & - & 1 & 3 \\
\hline & Instagram & - & - & - & - & - & - & - & - & - \\
\hline \multirow{3}{*}{$\begin{array}{l}\text { Earth Asso- } \\
\text { ciation }\end{array}$} & Facebook & - & - & - & - & 1 & - & - & - & 1 \\
\hline & Twitter & - & - & - & - & - & - & - & 2 & 2 \\
\hline & Instagram & - & - & - & - & - & - & - & - & - \\
\hline \multirow{3}{*}{$\begin{array}{l}\text { Mediterra- } \\
\text { nean Pro- } \\
\text { tection As- } \\
\text { sociation }\end{array}$} & Facebook & - & 1 & - & - & 1 & - & - & 1 & 3 \\
\hline & Twitter & - & 2 & - & 1 & 2 & - & - & 2 & 7 \\
\hline & Instagram & - & 2 & - & - & 1 & - & - & 1 & 4 \\
\hline \multirow{3}{*}{$\begin{array}{l}\text { Environ- } \\
\text { mental En- } \\
\text { ergy Asso- } \\
\text { ciation }\end{array}$} & Facebook & - & 1 & - & - & 1 & - & 2 & 1 & 5 \\
\hline & Twitter & - & 1 & - & 3 & 1 & - & 3 & 3 & 11 \\
\hline & Instagram & - & 1 & - & - & 1 & - & - & - & 2 \\
\hline \multirow{3}{*}{$\begin{array}{l}\text { Nature As- } \\
\text { sociation }\end{array}$} & Facebook & 1 & 1 & 1 & 1 & 1 & - & 1 & - & 6 \\
\hline & Twitter & 1 & 1 & 1 & 2 & - & - & 3 & - & 8 \\
\hline & Instagram & 1 & 1 & 1 & 1 & 1 & - & 1 & - & 6 \\
\hline \multirow{3}{*}{$\begin{array}{l}\text { Zeytince } \\
\text { Association }\end{array}$} & Facebook & - & 1 & - & - & - & - & - & - & 1 \\
\hline & Twitter & - & 1 & - & - & - & - & - & - & 1 \\
\hline & Instagram & - & 1 & - & - & - & - & - & - & 1 \\
\hline \multirow{5}{*}{$\begin{array}{l}\text { KOHAY- } \\
\text { DER Asso- } \\
\text { ciation }\end{array}$} & Facebook & 2 & 6 & 6 & 3 & 6 & 3 & 2 & 7 & 35 \\
\hline & Twitter & - & 2 & - & - & - & 1 & - & - & 3 \\
\hline & Instagram & 3 & 5 & 3 & 3 & 6 & 6 & 2 & 7 & 35 \\
\hline & Facebook & - & - & - & - & - & 1 & - & - & 1 \\
\hline & Twitter & - & - & - & - & - & 1 & - & - & 1 \\
\hline
\end{tabular}




\begin{tabular}{|c|c|c|c|c|c|c|c|c|c|c|}
\hline $\begin{array}{l}\text { Hemşin } \\
\text { Life Associ- } \\
\text { ation }\end{array}$ & Instagram & - & - & - & - & - & 1 & - & - & 1 \\
\hline \multirow{3}{*}{$\begin{array}{l}\text { TíB Federa- } \\
\text { tion }\end{array}$} & Facebook & - & 1 & - & 1 & - & - & - & - & 2 \\
\hline & Twitter & - & - & - & 2 & - & - & 1 & - & 3 \\
\hline & Instagram & - & 2 & - & 1 & - & - & 1 & - & 4 \\
\hline \multirow{3}{*}{$\begin{array}{l}\text { DOHAS } \\
\text { Association }\end{array}$} & Facebook & 3 & 3 & 4 & 4 & 5 & 7 & 6 & 2 & 34 \\
\hline & Twitter & - & - & 2 & - & 1 & 3 & 2 & - & 8 \\
\hline & Instagram & 2 & 3 & 1 & 3 & 3 & 4 & 3 & 2 & 21 \\
\hline
\end{tabular}

Table 4 depicts the number of Facebook, Twitter and Instagram shares of the organizations. The organizations with the highest number of shares on Facebook social network site during the week of June 4--11, 2019 were identified to be DOSDER Association with 42 posts, KOHAYDER Association with 35 shares and DOHAS Association with 34 shares. The organizations with no shares during the present study were TÜÇEV Foundation, Disaster Awareness Association, SURGED Association, Pan Association and Street is Ours Association. Considering the number of shares made on the basis of June 5, 2019 World Environment Day, 16 (45.7\%) of the organizations did not post at all that day. The ones that made the most shares on June 5, 2019 were KOHAYDER Association with 6 shares and DOSDER Association with 5 shares. The last sharing dates of the organizations with no shares (shown in Table 2) suggest that they did not make any sharing recently except for Street is Ours Association.

The effectiveness of word of mouth communication, which is among Hoffman and Fodor's metrics for measuring social media marketing effectiveness, is measured by the number of shares and posts. High number of shares made by DOSDER Association, KOHAYDER Association and DOHAS Association means that they stand out more in word-of-mouth communication and they are successful. Besides, they could be said to use social media effectively in sharing and updating posts on a daily basis and on 4-11 June 2019.

Table 4 presents that the organizations sharing the most posts from Twitter accounts were TEMA Foundation, WWF Foundation, DOĞÇEV Association and Environmentalist Energy Association. TÜÇEV Foundation has not yet shared any content since it created Twitter account. Table 
4 also suggests that organizations generally made fewer sharings on Twitter accounts than Facebook and Instagram social network sites while this study was being carried out. They used Twitter social network site less actively than the other two social networking sites. TEMA Foundation and WWF Foundation were the only ones sharing content every day during the present study, meaning that these two organizations use Twitter effectively and actively. In addition, DOSDER Association, which made the most shares on Facebook, did not share any content from its Twitter account throughout the study, while KOHAYDER Association and DOHAS Association made fewer share contents compared to the posts in their Facebook accounts.

According to Table 4, the organizations that made the most shares from their Instagram accounts were KOHAYDER Association with 35 shares, DOHAS Association with 21 shares, TEMA Foundation and WWF Foundation with 11 shares. The TEMA Foundation and WWF Foundation, having the highest number of followers / members on the Instagram social network, can be defined as organizations with brand awareness and brand engagement. In addition, the number of likes is taken as a basis to measure the effectiveness of word-of-mouth communication. In this vein, TEMA Foundation and WWF Foundation can be said to be successful in wordof-mouth communication due to the number of likes for the content they have shared on their Instagram accounts.

Although KOHAYDER Association and DOHAS Association made more shares during the study week (4-11 June 2019), TEMA Foundation and WWF Foundation were more successful in word-of-mouth communication. This may be due to the high number of followers and reaching more people. TEMA Foundation received 47,008 likes, WWF Foundation 27,046 likes, KOHAYDER Association 1,088 likes and DOHAS Association 975 likes during the study. What is more, brand engagement can be measured depending on the number of comments received. The WWF Foundation received a total of 2,499 comments throughout the study, referring to the fact that users have brand engagement towards the foundation. 


\section{Result}

This study attempts to identify whether social media, which is used by organizations whose popularity is increasing day by day in order to communicate with the target audience, is also used effectively by the nonprofit organizations. The aim of the study is to determine whether the organizations identified within the scope of the sample use Facebook, Twitter and Instagram effectively in order to increase their brand awareness, create engagement to their brands and provide word-of-mouth communication.

The results obtained according to Hoffman and Fodor' metrics related to social media marketing effectiveness measurement are as follows;

Organizations with high brand awareness by taking the number of followers / members / fans as a criterion for Facebook social media site were determined as TEMA Foundation, WWF Foundation, Nature Association, TEKFEN Foundation and ÇEVKO Foundation. Those with the highest brand engagement based on the number of likes and comments were noted as TEMA Foundation and WWF Foundation. DOSDER Association, KOHAYDER Association, DOHAS Association, DOĞÇEV Association and Natural Life Association were successful in word-of-mouth communication by taking the number of shares and posts as a criterion. TEMA Foundation was most talked about organization on "June 5, 2019 Environment Day". Organizations with the most followers were TEMA Foundation and ÇEVKO Foundation, while those losing the most followers were WWF Foundation and Nature Association. The organization that received the most engagement was TEMA Foundation. The most shared type of content by organizations was identified as photos. Users, on the other hand, mostly used "like", one of the engagement types. This was followed by "comment" and "reshare the shared content ".

Based upon the number of followers for the Twitter social media site, the organizations with high brand awareness and brand engagement were TEMA Foundation, WWF Foundation, Nature Association, Earth Association and ÇEKUD Association. Taking the number of retweets as a criterion, the organizations that were successful in word-of-mouth communication and the most talked about organizations were TEMA Foundation and ÇEKUD Association. Besides, those with the most followers were 
TEMA Foundation, WWF Foundation, Nature Association, Earth Association and Mind Your Waste Foundation; whereas those losing the most followers were ECORDING Association, DOHAS Association, Street is Ours Association, DOĞÇEV Association and SURGED Association. In addition, the organizations that received the most engagement were TEMA Foundation and WWF Foundation. The most shared type of content by organizations was photos. Users mostly used "like" as one of the engagement types, which was followed by "retweet".

Organizations with high brand engagement based on the number of followers for the Instagram social media site were noted as TEMA Foundation, WWF Foundation, Nature Association, TEKFEN Foundation and Mind Your Waste Foundation. Those which were successful in word-ofmouth communication and which were the most talked about were TEMA Foundation and WWF Foundation. Organizations with the most followers were TEMA Foundation, ÇEVKO Foundation, Earth Association, TURMEPA Association and Mind Your Waste Foundation, while those losing the most followers were ECORDING Association, DOHAS Association, ASPEG Association, Pan Association and Boğaziçi Patililer Association. The organizations that received the most engagement were TEMA Foundation and WWF Foundation. The most shared type of content by organizations was identified as photos. Users mostly used "like", one of the engagement types.

As a conclusion, this study aims to determine whether non-profit organizations operating in the field of environmental protection use Facebook, Twitter and Instagram social media sites as an effective communication channel while reaching their target audiences. Based upon the results, it is likely to say that the organizations used social media tools effectively and actively, especially about TEMA Foundation and WWF Foundation. Similar studies were conducted on this subject. For instance; in his master's thesis on comparing Facebook accounts of TEMA Foundation and TURMEPA Clean Sea Association through using content analysis method, Aydin (2019, p.51-88-91) concluded that TEMA Foundation had much more interaction than the other compared organizations and that they actively used social media. The researcher also stated that the WWF Foundation was one of the organizations that actively uses social networking sites such as Facebook and Twitter, which provide high speed and low 
cost in informing and promoting, publishing advertisements, gaining awareness, interpersonal communication and gaining volunteers. These results are parallel to those of our study. In another study, Lovejoy and Saxton (2012, p.340-349) examined the Twitter accounts of various nonprofit organizations, including environmental organizations, over onemonth period. The results showed that the majority of organizations actively used social media. Novak (2012, p.86), in the master's thesis, aimed to identify whether non-profit organizations used social media effectively as a marketing tool or not. Accordingly, non-profit organizations were found to use social media accounts more effectively to interact with their followers than for-profit organizations. Similar studies are available in the relevant literature and the samples can be augmented. In conclusion, social networking sites can be an effective way for non-profit organizations to promote themselves, gain awareness, and strengthen word of mouth by interacting with their followers. At that point, TEMA Foundation and WWF Foundation may be said to actively use social network sites for these purposes.

In brief, TEMA Foundation and WWF Foundation are thought to be the first prominent organizations in the field of Environment and Natural Life Protection. This study concluded that TEMA Foundation and the WWF Foundation are known and recognized on the social media sites, and they have gained customer engagement and become successful in word of mouth communication.

\section{References}

Aydın, B. (2019). Sivil toplum kuruluşlarının sosyal medya içeriklerinde gönülliülük faaliyetlerinin değerlendirilmesi: Deniztemiz Derneği örneği. Yüksek Lisans Tezi. İstanbul. İstanbul Üniversitesi.

Barutçu, S. and Tomaş, M. (2013). Sürdürülebilir sosyal medya pazarlaması ve sosyal medya pazarlaması etkinliğinin ölçümü. İnternet Uygulamalan ve Yönetimi Dergisi, 4(1), 5-23.

Brown, S. (2012). Social information: Gaining competitive and business advantage using social media tools. Oxford: Chandos Publishing http://web.a.ebscohost.com/ehost/ebookviewer/ebook?sid=c8ecd2cc-a220-4b2a-b734- 
705b9fd39e81\%40sdc-v-sessmgr02\&vid=7\&rid=21\&format=EB adresinden alındı. Erişim tarihi 15 Ekim 2019

Cray, E. (2012). Successful social media measurement from an agency standpoint. The Elon Journal of Undergraduate Research in Communications, 3(1), 43-51.

Ghali, P. (2011). Calculating your social media marketing return on investment : A how-to guide for new social media marketers, 1-14. https://www.academia.edu/7797170/Calculating_Social_Media_ROI adresinden alındı. Erişim tarihi 14 Kasım 2019

Hoffman, D. L. and Fodor, M. (2010). Can you measure the ROI of your social media marketing? MITSLOAN Management Review, 52(1), 40-50.

Kaçar, A. İ. (2014). Sosyal pazarlama ve kampanya yönetiminin çevreci sivil toplum kuruluşlar açısından incelenmesi: Karma bir araştırma. Doktora tezi. Manisa: Celal Bayar Üniversitesi.

Lovejoy, K., and Saxton, G. D. (2012). Information, community, and action: How nonprofit organizations use social media. Journal of Computer-Mediated Communication, 7, 337-353.

Luo, Y. and Jiang, H. (2012). A dialogue with social media experts: Measurement and challenges of social media use in Chinese public relations practice. Global Media Journal, 5(2), 57-74.

Nurtanış Velioğlu, M. and Çoknaz, D. (2010). Sosyal pazarlama. Varinli İ. and Çatı K. (Ed.), Güncel Pazarlama Yaklaşımlarından Seçmeler içinde (p.455-478). Ankara: Detay Yayıncllı.

Novak, M. (2012). Consumer perception of the efficacy of social media branding by non-profit and for-profit organizations. Yüksek Lisans Tezi. Roman University.

Pencarelli, T. and Mele, M.G. (2019). A systematic literature review on social media metrics. Mercati \& Competitive, 1, 15-38.

Saatçioğlu, E. (2017). Sivil toplum örgütlerinin sosyal medya kullanımları: Greenpeace Türkiye facebook sayfası örneği. Selçuk İletişim, 10(1), 158-187.

Wendlandt, L. B. (2012). Return on investment concerns in social media marketing:

An examination of recent cases. Yüksek Lisans Tezi. Master's of Business Administration and the Master's of Arts in Management, The College of St. Scholastica, Duluth, $\mathrm{MN}$.

Yılmazel, M. (2011). Türkiye'de faaliyet gösteren vakuflarm sosyal medya kullanmmlarna yönelik bir içerik analizi. Yüksek Lisans Tezi. Eskişehir: Anadolu Üniversitesi.

Zeren, D. (2015). Örneklem seçimi. Demrici Orel, F.(Ed.), Pazarlama Araştırması içinde (p.208-235). Ankara:Nobel Yayıncllik

https://wwww.boomsocial.com/. (n.d.). Erişim tarihi 12 Ekim 2019 
https://www.siviltoplum.gov.trl. (n.d.). Erişim tarihi 28 Mayıs 2019

https://www.socialbakers.com/blog/7-steps-to-measure-social-media-marketing-roi-a-copleteguide. (2018, Şubat 23). Erişim tarihi 13 Kasım 2019

https://www.vgm.gov.tr. (n.d.). Erişim tarihi 28 Mayıs 2019

\section{Kaynakça Bilgisi / Citation Information}

Yalçın, Z. and Canoğlu, M. (2021). Social Media Marketing Effectiveness of Non-Profit Organizations: An Analysis on Environmentalist Organizations. OPUS- International Journal of Society Studies, 18(Yönetim ve organizasyon Özel Sayıs1), 1136-1163. DOI: 10.26466/opus. 918660 